\title{
Cortical Thickness and Serum NfL Explain Cognitive Dysfunction in Newly Diagnosed Patients With Multiple Sclerosis
}

Álvaro J. Cruz-Gomez, PhD, Lucía Forero, MD, Elena Lozano-Soto, BSc, Fátima Cano-Cano, BSc, Florencia Sanmartino, BSc, Raúl Rashid-López, MD, Jsé Paz-Expósito, MD, PhD, Jaime D. Gómez Ramirez, PhD, Raúl Espinosa-Rosso, MD, and Javier J. González-Rosa, PhD

Neurol Neuroimmunol Neuroinflamm 2021;8:e1074. doi:10.1212/NXI.0000000000001074

\section{Abstract}

\section{Background and Objectives}

To determine the relative importance of global or regional MRI and blood markers of neurodegeneration and neuroaxonal injury in predicting cognitive performance for recently diagnosed patients with multiple sclerosis (MS).

\section{Methods}

Thirty-five newly diagnosed patients with relapsing-remitting MS (RRMS) and 23 healthy controls (HCs) simultaneously completed a full clinical and neuropsychological assessment, structural brain MRI, and serum neurofilament light chain ( $\mathrm{sNfL}$ ) level test. Linear regression analyses were performed to determine which global or regional measures of gray matter (GM) atrophy and cortical thickness (CT), in combination with sNfL levels and clinical scores, are most strongly related to neuropsychological impairment.

\section{Results}

Compared with HCs, patients with MS showed bilateral thalamic GM atrophy (left, $p=0.033$; right, $p=0.047)$ and diminished CT, particularly in the right superior and transverse temporal gyri $(p=0.045 ; p=0.037)$. Regional atrophy failed to add predictive variance, whereas anxiety symptoms, sNfL, and global CT were the best predictors $\left(\mathrm{R}^{2}=0.404 ; p<0.001\right)$ of cognitive outcomes, with temporal thickness accounting for greater variance in cognitive deficits than global CT.

\section{Discussion}

Thalamic GM atrophy and thinning in temporal regions represent a distinctive MRI trait in the early stages of MS. Although sNfL levels alone do not clearly differentiate HCs and patients with RRMS, in combination with global and regional CT, sNfL levels can better explain the presence of underlying cognitive deficits. Hence, cortical thinning and sNfL increases can be considered 2 parallel neurodegenerative markers in the pathogenesis of progression in newly diagnosed patients with MS.
Correspondence

Dr. González-Rosa javier.rosa@uca.es

From the Institute of Biomedical Research and Innovation of Cadiz (INiBICA) (A.J.C.-G., L.F., E.L.-S., F.C.-C., F.S., R.R.-L., J.D.G.R., R.E.-R., J.J.G.-R.), Cadiz, Spain; Psychology Department (A.J.C.-G., E.L.-S., F.S., J.D.G.R., J.J.G.-R.), University of Cadiz, Spain; Neurology Department (L.F., R.R.-L., R.E.-R.), Puerta del Mar University Hospital, Cadiz, Spain; and Radiodiagnostic Department (J.P.-E.), Puerta del Mar Hospital, Cadiz, Spain. 


\section{Glossary}

BDI = Beck Depression Inventory; BPF = brain parenchymal fraction; BRB-N = Brief Repeatable Battery of Neuropsychological Tests; CAT = Computation Anatomy Toolbox; CI = cognitively impaired; CP = cognitively preserved; CT = cortical thickness; EDSS = Expanded Disability Status Scale; FDR = false discovery rate; FIRST = FMRIB's Integrated Registration and Segmentation Tool; FLAIR = fluid-attenuated inversion recovery; FSS = fatigue severity scale; $\mathbf{G M}=$ gray matter; GMF = gray matter fraction; HC = healthy control; IFOG = inferior frontal orbital frontal gyrus; $\mathbf{M S}=$ multiple sclerosis; NI = neuropsychological impairment; ROI = regions of interest; RRMS = relapsing-remitting multiple sclerosis; SDMT = Symbol Digit Modalities Test; secondary progressive MS; sNfL = serum neurofilament light chain; SP = secondary progressive; $\mathbf{S R T}=$ Selective Reminding Test; STAI = State-Trait Anxiety Inventory; TTG = transverse temporal gyrus; $\mathbf{W M}=$ white matter; ZG = global cognitive Z score.

Neuroimaging studies using MRI have reported associations between the severity of cognitive dysfunction in multiple sclerosis (MS) and structural measures of neuronal damage, highlighting gray matter (GM) atrophy as one of the best radiologic predictors of cognitive impairment severity and development. ${ }^{1}$ In this regard, several studies have revealed that subcortical GM atrophy is already detectable at initial disease stages and is strongly correlated with MS patients' clinical and cognitive status. ${ }^{2,3}$

Cortical atrophy has also been suggested to represent a common phenomenon in the course of MS, and recent evidence supports its association with cognitive dysfunction, especially when surface-based measures such as cortical thickness (CT) are used rather than traditional volume-based measures. ${ }^{4-6}$ Although both measures are correlated between them, they may provide complementary information regarding cortical structure, reflecting differential GM morphologic alterations. ${ }^{7}$ Nevertheless, studies addressing the regional effects of CT on cognitive function are limited, especially in mildly disabled patients with relapsing-remitting MS (RRMS).

More recently, the concentration of serum neurofilament light chain (sNfL), a component of the axonal cytoskeleton essential to maintain the structural integrity of neuronal cells, has sparked considerable interest as a blood-based biomarker to easily detect neurodegeneration and monitor disease progression in different neurologic diseases ${ }^{8-11}$, including MS. ${ }^{12,13}$ Within this framework, an increasing number of studies have demonstrated that compared with healthy populations, patients with MS exhibit higher sNfL concentrations, which are also associated with clinical disability and radiologic measures of disease activity, such as brain atrophy and lesion load. ${ }^{13-15}$

Unfortunately, to date, studies assessing the direct relationship between sNfL levels and cognitive function in MS are scarce or have only included patients with long-standing and progressive $\mathrm{MS}^{16}$; therefore, the role of sNfL levels as an alternate biomarker for cognitive status remains controversial. ${ }^{17}$ However, recent longitudinal studies have demonstrated that higher sNfL levels are associated with the development of brain atrophy in different neurologic diseases, ${ }^{10,18}$ a finding that points to a close relationship between increased brain atrophy resulting from ongoing axonal loss and higher sNfL levels and highlights the importance of the changes in this relationship over time.

Although the direct involvement of GM volume and CT in cognitive dysfunction in MS has been suggested, it remains elusive how sNfL concentration as a measure of neuroaxonal injury could ultimately contribute to this cognitive decline. In the present study, the specific contributions of clinical and psychiatric assessment data, of well-established MRI-based global and regional measures reflecting macrostructural damage, and of a biological marker of neuroaxonal damage (i.e., sNfL levels) in explaining cognitive abnormalities in newly diagnosed and slightly disabled patients with MS were investigated. First, we explore whether global MRI measures of atrophy and cortical thinning, together with $\mathrm{sNfL}$ and clinical and psychiatric variables, explained cognitive dysfunction in patients with MS. Second, we asked whether, replacing these global MRI measures, regional-specific MRI measures of atrophy and cortical thinning accounted for additional predictive variance of cognitive abnormalities. Establishing a joint model between subcortical GM atrophy, CT, and sNfL levels would provide updated brain structural biomarkers underlying cognitive dysfunction at the initial stages of MS.

\section{Methods}

\section{Study Participants}

Thirty-five patients diagnosed with clinically definite RRMS according to the McDonald criteria ${ }^{19}$ were recruited from the Hospital Universitario Puerta del Mar of Cadiz. Patients with RRMS were enrolled in this cross-sectional study based on the following criteria: (1) aged between 18 and 58 years; (2) having been diagnosed with MS within 4 years; (3) having a mild physical disability (Expanded Disability Status Scale [EDDS] score equal to or less than 3.5); and (4) relapse and steroid free for at least 2 months before the study. In addition, 23 participants with no history of neurologic or psychiatric dysfunction were included as healthy controls (HCs). Handedness was assessed by the Edinburgh Handedness Inventory. ${ }^{20}$ Clinical and neuropsychological measures, serum sampling, and MRI were all performed in 2 separate sessions (months between tests: $3.4 \pm 3.1$ ). 
Table 1 Demographic and Clinical Characteristics of Participants

\begin{tabular}{|c|c|c|c|}
\hline Variables & RRMS & $\mathrm{HC}$ & HC vs RRMS ( $p$ values) \\
\hline $\mathbf{N}$ & 35 & 23 & - \\
\hline Age & $38.40(10.24)$ [18-57] & 35.43 (7.15), [25-48] & 0.232 \\
\hline \multicolumn{4}{|l|}{ Sex } \\
\hline Male/female & $15 / 20$ & $10 / 13$ & 0.963 \\
\hline$\%$ Female & $57.14 \%$ & $56.52 \%$ & - \\
\hline Education y & 13.06 (3.20) [6-20] & 17.87 (2.49) [15-22] & $0.000^{\mathrm{a}}$ \\
\hline EDSS median & $1.0(1.27)[0-3.5]$ & & \\
\hline \multicolumn{4}{|l|}{ 9HPT (s) } \\
\hline Dominant hand & $24.84(4.69)$ [15.06-36.05] & - & - \\
\hline Nondominant hand & $27.26(8.37)$ [17.80-55.10] & - & - \\
\hline T25FW (s) & $8.92(1.93)[6.05-16.02]$ & & \\
\hline Disease duration (y) & $3.13(1.62)[0-5]$ & & \\
\hline \multicolumn{4}{|l|}{ DMT } \\
\hline None (\%) & $5.71 \%$ & - & - \\
\hline First line (\%) & $51.43 \%$ & - & - \\
\hline Second line (\%) & $42.86 \%$ & - & - \\
\hline \multicolumn{4}{|c|}{$\begin{array}{l}\text { Abbreviations: DMT = disease-modifying therapy; EDSS }=\text { Expanded Disability Status Scale; HC }=\text { healthy control; RRMS }=\text { relapsing-remitting multiple } \\
\text { sclerosis; T25FW }=\text { timed } 25 \text {-foot walk; } 9 \mathrm{HPT}=9 \mathrm{Hole} \text { Peg Test; }(1) \text { first-line modifying treatment: glatiramer acetate/interferons/dimethyl fumarate; }(2) \\
\text { second-line modifying treatment: natalizumab/fingolimod. } \\
\text { Values are expressed as the mean and SD and [range]. Student } t \text { tests for continuous variables and } \chi^{2} \text { for categorical variables were performed to assess } \\
\text { significant differences between groups ( } p \text { values are included). } \\
\text { a Significantly different at } p<0.01 \text {. }\end{array}$} \\
\hline
\end{tabular}

\section{Standard Protocol Approvals, Registrations, and Patient Consents}

The protocol for the present study was approved by the Andalusian Biomedical Research Ethics Committee (Ref.: LFD-VIT2018-01). All participants provided informed written consent before participation in accordance with the Declaration of Helsinki.

\section{Neurologic Examination}

Patients selected for the present study underwent a neurologic assessment that included the EDSS, ${ }^{21}$ timed 25-foot walk test, and 9-hole peg test. Clinical and demographic information on patient characteristics and prescribed diseasemodifying therapy was noted for all patients.

\section{Neuropsychological Assessment}

All participants were assessed using (1) the Brief Repeatable Battery of Neuropsychological Tests (BRB-N), validated for the Spanish population ${ }^{22}$; (2) the Matrix Reasoning Subtest of the Wechsler Adult Intelligence Scale (III) to determine the $\mathrm{IQ}^{23}$; (3) the Fatigue Severity Scale (FSS) ${ }^{24}$; (4) the Beck Depression Inventory (BDI ${ }^{25}$; and (5) the State-Trait Anxiety Inventory $(\mathrm{STAI}){ }^{26}$

For each subtest of the BRB-N, Z scores were calculated for each patient using the mean and SD obtained for the HC group
( $\mathrm{Z}$ score $=$ Raw score $-\mathrm{HC}$ mean/HC SD). Subsequently, a global cognitive $Z(Z G)$ score was obtained for each participant, averaging $9 \mathrm{Z}$ scores from each subtest of the BRB-N.

\section{sNfL Level Analysis}

Blood samples were collected in clot-activating serum separator tubes, centrifuged for 10 minutes at $1,500 \mathrm{~g}$ to separate serum and properly stored at $-80^{\circ} \mathrm{C}$. The resulting serum was aliquoted and properly stored at $-80^{\circ} \mathrm{C}$ until analysis. sNfL concentrations were measured using a new high-sensitivity sandwich ELISA method for human samples (Aviva Systems Biology, San Diego) according to the manufacturer's instructions. All patient samples were appropriately diluted to fall in the range of the standard curve, whereas control samples were not diluted. The abovementioned method has been previously validated and is described in detail elsewhere. ${ }^{8}$

\section{MRI Data Acquisition}

For all subjects, brain MRIs were collected using a $1.5 \mathrm{~T}$ scanner (Philips Medical System, Ingenia CX, Best, Netherlands). The following sequences were acquired: (1) sagittal T1weighted 3D magnetization prepared rapid acquisition gradient echo (MPRAGE) sequence (repetition time $[\mathrm{TR}]=2.200 \mathrm{~ms}$; echo time $[\mathrm{TE}]=3 \mathrm{~ms}$; flip angle $=15^{\circ}$, matrix $=256 \times 256 \times$ 160 , and voxel size $=1 \times 1 \times 1 \mathrm{~mm})$ and $(2)$ sagittal fluidattenuated inversion recovery (FLAIR) 3D sequence $(\mathrm{TR}=$ 
Table 2 Neuropsychological and Global MRI Characteristics of Participants

\begin{tabular}{|c|c|c|c|}
\hline Variables & RRMS & $\mathrm{HC}$ & HC vs RRMS ( $p$ values) \\
\hline \multicolumn{4}{|l|}{ Manipulative IQ } \\
\hline Matrix design (WAIS-III) & $103.57(14.07)$ & $110.22(12.66)$ & 0.073 \\
\hline \multicolumn{4}{|l|}{ Fatigue severity } \\
\hline FSS & $42.60(14.73)$ & $26.22(11.35)$ & $0.000^{\mathrm{a}}$ \\
\hline \multicolumn{4}{|l|}{ Depression } \\
\hline BDI-II & $17.17(10.24)$ & $7.04(5.90)$ & $0.000^{\mathrm{a}}$ \\
\hline \multicolumn{4}{|l|}{ Anxiety } \\
\hline STAI-state & $24.60(12.58)$ & $18.09(9.93)$ & $0.041^{b}$ \\
\hline STAI-trait & $28.74(14.23)$ & $19.30(11.58)$ & $0.010^{\mathrm{b}}$ \\
\hline \multicolumn{4}{|l|}{ BRB-N } \\
\hline SDMT & $55.37(14.24)$ & $63.00(13.97)$ & 0.110 \\
\hline PASAT $3 \mathrm{~s}$ & $40.86(11.73)$ & $48.09(8.73)$ & $0.017^{b}$ \\
\hline SRT long-term storage & $48.63(10.81)$ & $56.04(9.67)$ & $0.001^{a}$ \\
\hline SRT consistent long-term retrieval & $41.09(13.31)$ & $49.78(12.29)$ & $0.010^{\mathrm{b}}$ \\
\hline SRT delayed recall & $8.91(2.34)$ & $10.22(1.68)$ & $0.009^{a}$ \\
\hline 10/36 SPART long-term storage & $19.83(6.22)$ & $22.30(3.89)$ & 0.147 \\
\hline 10/36 SPART delayed recall & $6.89(2.85)$ & $8.09(1.90)$ & 0.105 \\
\hline Phonetic Fluency (F) & $11.23(4.63)$ & $13.00(3.28)$ & 0.146 \\
\hline Semantic Fluency (animals) & $20.66(5.57)$ & $23.70(4.55)$ & 0.053 \\
\hline \multicolumn{4}{|l|}{ MRI global measures } \\
\hline FLAIR WM lesion volume (mL) & $4.02(5.88)$ & - & - \\
\hline BPF & $0.79(0.04)$ & $0.81(0.03)$ & 0.167 \\
\hline GMF & $0.44(0.31)$ & $0.45(0.25)$ & 0.315 \\
\hline Global cortical thickness (mm) & $2.65(0.11)$ & $2.73(0.10)$ & $0.010^{\mathrm{b}}$ \\
\hline \multicolumn{4}{|l|}{ Serum neurofilament light levels } \\
\hline sNfL (pg/mL) & $44.48(27.70)$ & $33.28(16.47)$ & 0.087 \\
\hline sNfL (median, interquartile range) & $38(31-51)$ & $36(20-42)$ & - \\
\hline
\end{tabular}

Abbreviations: BDI = Beck Depression Inventory; BPF = brain parenchymal fraction; BRB-N = Brief Repeatable Battery of Neuropsychological Tests; FSS = Fatigue Severity Scale; GMF = gray matter fraction; HC = healthy control; PASAT = Paced Auditory Serial Addition Test; RRMS = relapsing-remitting multiple sclerosis; SDMT = Symbol Digit Modalities Test; SNfL = serum neurofilament light; SPART = Spatial Recall Test; SRT = Selective Reminding Test; STAI = State-Trait Anxiety Inventory; WAIS = Wechsler Adult Intelligence Scale; WM = white matter.

Values are expressed as the mean and SD. Student $t$ tests for continuous variables were performed to assess significant differences between groups ( $p$ values are included). Analysis of covariance design was used to assess BRB-N score comparisons including educational level was included as a nuisance covariate. a Significantly different at $p<0.01$.

${ }^{\mathrm{b}}$ Significantly different at $p<0.05$.

$6,000 \mathrm{~ms} ; \mathrm{TE}=354 \mathrm{~ms}$; flip angle $=180^{\circ}$, matrix $=196 \times 256 \times$ 160 , and voxel size $=1.05 \times 1.05 \times 1 \mathrm{~mm}$ ).

\section{MRI Data Processing}

\section{Quantification of WM Lesion Volumes}

In RRMS patient MRI scans, FLAIR hyperintense white matter (WM) lesions were automatically segmented and quantified in milliliters using the Lesion Segmentation Tool with the lesion growth algorithm (applied-statistics.de/lst.html). To improve the tissue segmentation step in subsequent image preprocessing for GM volume assessments, ${ }^{27} \mathrm{~T} 1$ hypointense lesions were also identified and filled using neighboring WM values in RRMS images using the same toolbox.

\section{Quantification of Global and Regional GM Volumes}

FMRIB's Integrated Registration and Segmentation Tool (fmrib.ox.ac.uk/fsl/first/), which is part of the FMRIB 
Table 3 Subcortical GM Volumes in Cubic Millimeters Obtained Using FIRST in Each Experimental Group and Significant Differences Between Groups Corrected for Total Brain Volume

\begin{tabular}{llll}
\hline Variables & RRMS & HC & HC vs RRMS $(\boldsymbol{p}$ values $)$ \\
\hline Left thalamus & $5511.72(620.88)$ & $6063.18(568.41)$ & $0.033^{\mathrm{a}}$ \\
\hline Right thalamus & $5346.37(625.33)$ & $5859.15(507.12)$ & $0.047^{\mathrm{a}}$ \\
\hline Left accumbens & $300.48(86.34)$ & $294.17(109.43)$ & 0.132 \\
\hline Right accumbens & $224.55(71.75)$ & $220.29(90.17)$ & 0.120 \\
\hline Left caudate & $2351.51(426.41)$ & $2602.04(271.12)$ & 0.233 \\
\hline Right caudate & $2472.38(388.14)$ & $2616.60(391.62)$ & 0.703 \\
\hline Left pallidum & $941.83(126.96)$ & $918.34(194.67)$ & 0.387 \\
\hline Right pallidum & $914.81(111.33)$ & $911.68(198.55)$ & 0.917 \\
\hline Left putamen & $2793.01(495.57)$ & $2884.03(369.29)$ & 0.238 \\
\hline Right putamen & $2765.79(498.14)$ & $2851.86(330.08)$ & 0.278 \\
\hline Left hippocampus & $2711.39(395.86)$ & $2824.25(473.94)$ & 0.769 \\
\hline Right hippocampus & $2613.36(358.7)$ & $2692.41(382.51)$ & $1017.21(186.52)$ \\
\hline Left amygdala & $900.72(159.74)$ & $911.12(225.17)$ & 0.812 \\
\hline Right amygdala & $808.81(222.3)$ & 0.075 & 0.287 \\
\hline
\end{tabular}

Abbreviations: FIRST = FMRIB's Integrated Registration and Segmentation Tool; GM = gray matter; HC = healthy control; RRMS = relapsing-remitting multiple sclerosis.

Values are expressed as the means and SD. ANCOVA design following a general linear model was used to compare subcortical GM volumes between groups and including total brain volume was included as a nuisance covariate in all comparisons ( $p$ values are included).

a Significantly different at $p<0.05$.

Software Library, was used to obtain regional GM volumes of subcortical structures (including the caudate, putamen, globus pallidus, thalamus, hippocampus, amygdala, and nucleus accumbens) and subsequently corrected for brain volume. $^{28}$

Voxel-based morphometry analysis was performed using the Computation Anatomy Toolbox (CAT-12, version 12.6, r1450) with the current version of Statistical Parametric Mapping (12; fil.ion.ucl.ac.uk/spm/). To this end, T1-3D images were first visually inspected for artifacts and then preprocessed following standard steps suggested in the CAT-12 manual, including (1) bias-field correction; (2) segmentation into GM, WM, and CSF; (3) registration to a standard template using the DARTEL algorithm; (4) normalization of GM images to the Montreal Neurological Institute template; and (5) modulation of the normalized data. GM regional volumes of cortical areas of interest were extracted using the Neuromorphometrics atlas (neuromorphometrics.com/) and corrected for total intracranial volume.

The brain parenchymal fraction (BPF) was determined through tissue-specific maps obtained in the segmentation step. The BPF was calculated by dividing the sum of GM and WM segmentation maps by the intracranial volume (sum of WM, GM, and CSF volumes) as an index of global atrophy. The GM fraction (GMF) was also obtained following the same procedure, dividing GM by intracranial volume, as an index of global specific GM atrophy.

\section{Quantification of Global and Regional CT Values}

CT was also assessed using the CAT- 12 toolbox a fully automated method that uses tissue segmentation to estimate distances between WM and GM voxels based on the projectionbased thickness method, ${ }^{29}$ including topology correction and spherical mapping. In addition to global CT measures, regional $\mathrm{CT}$ values were extracted using the Desikan-Killiany atlas. ${ }^{30}$

\section{Statistical Analysis}

SPSS 24.0 (IBM, Armonk, NY) was used to analyze clinical, neuropsychological, and global radiologic measures. After verifying the normal distribution of all continuous variables (Shapiro-Wilk test), analysis of variance, parametric Student $t$ test, and nonparametric Mann-Whitney $U$ test were performed as appropriate for continuous variables, and $\chi^{2}$ was used for categorical variables. Two analysis of covariance designs following a general linear model were used to compare neuropsychological scores and subcortical GM volumes between groups, including educational level and total brain volume as nuisance covariates, respectively. Regarding analyses of cortical regions of interest (ROI), significant differences between groups in volume and $\mathrm{CT}$ in areas defined by the atlases were assessed using the Analyze ROI function included in the CAT-12 
A. Deep gray matter volume

First segmentation

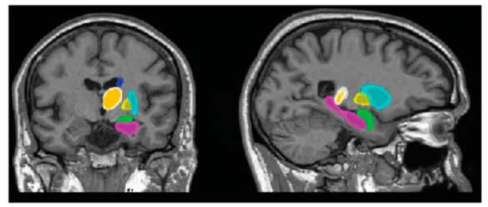

RRMS $<H C$
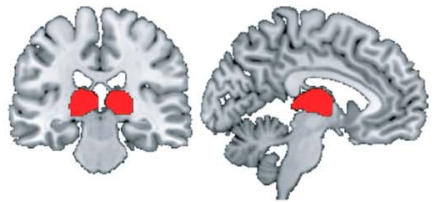

FDR/Holm-Bonferroni corrected $(p<0.05)$

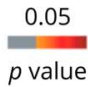

B. Cortical gray matter volume Neuromorphometrics atlas
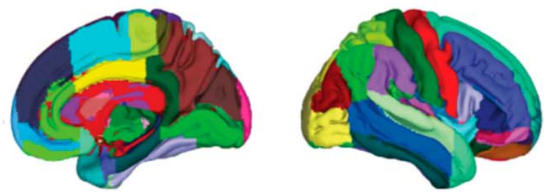

RRMS $<H C$

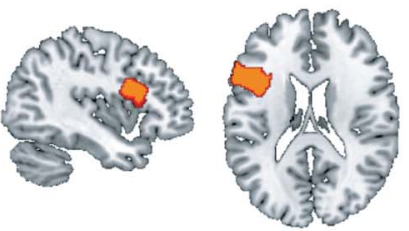

FDR corrected $(p<0.05)$

$p .05$

C. Cortical thickness

DK-40 atlas

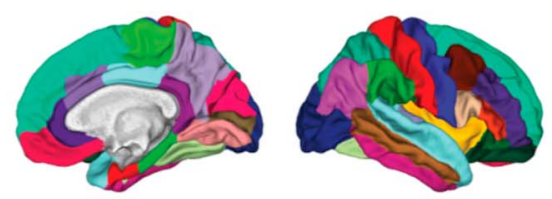

RRMS $<H C$

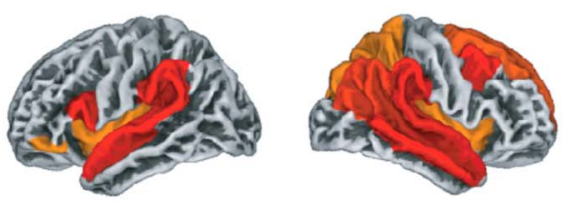

FDR corrected $(p<0.05)$

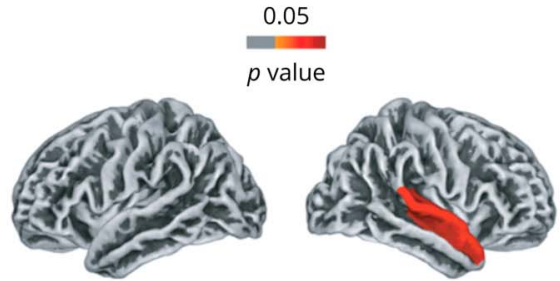

Holm-Bonferroni corrected $(p<0.05)$

(A) Example of FIRST deep GM structure segmentation in subject space. Compared with HCs, patients with RRMS showed a significant reduction in the left and right thalami. (B) Neuromorphometrics atlas used for cortical GM volume analysis in which, compared with HCs, patients with RRMS showed a significant reduction in the left inferior frontal orbitofrontal gyrus GM volume after application of FDR multiple comparison correction. After Holm-Bonferroni multiple comparison corrections, no significant effects were observed. (C) Desikan-Killiany atlas used for regional CT analysis in which patients with RRMS showed a significant reduction in CT in bilateral temporal, parietal, and frontal areas compared with HCS after FDR multiple comparison correction. After HolmBonferroni multiple comparison correction, patients with RRMS showed a significant reduction in CT only in the right TTG and right STG compared with HCS. $\mathrm{CT}=$ cortical thickness; FDR = false discovery rate; FIRST = FMRIB's Integrated Registration and Segmentation Tool; GM = gray matter; HC = healthy control; RRMS = relapsing-remitting multiple sclerosis; TTG = transverse temporal gyrus.

toolbox. Data were corrected for multiple comparisons using the false discovery rate (FDR) and Holm-Bonferroni methods.

Pearson and Spearman correlation analyses were performed to assess significant relationships between variables of interest (i.e., clinical, sNfL, and radiologic measures). In addition, to further evaluate the subsequent relationship between sNfL and cognitive status, patients with MS were split into 2 MS subgroups based on the presence of neuropsychological impairment (NI). The cutoff for failure in each neuropsychological test was defined here as a score below 1.5 SD from the mean score of the $\mathrm{HC}$ group, with NI then defined considering the number of failed 
Table 4 Significant Differences Between Regional GM Cortical Volumes and CT Measures in the Contrast of Healthy Controls > Patients With RRMS

\begin{tabular}{|c|c|c|}
\hline Variables & $\begin{array}{l}\text { HC > RRMS (FDR corrected) } \\
p \text { values }\end{array}$ & $\begin{array}{l}\text { HC > RRMS (Holm-Bonferroni corrected) } \\
p \text { values }\end{array}$ \\
\hline \multicolumn{3}{|c|}{ GM cortical regional volumes (normalized for brain volume) } \\
\hline Left inferior frontal orbital gyrus & 0.031 & n.s. \\
\hline \multicolumn{3}{|l|}{ CT regional values } \\
\hline Left transverse temporal gyrus & 0.026 & n.s. \\
\hline Right transverse temporal gyrus & 0.023 & 0.037 \\
\hline Left superior temporal gyrus & 0.026 & n.s. \\
\hline Right superior temporal gyrus & 0.023 & 0.045 \\
\hline Right middle temporal gyrus & 0.026 & n.s. \\
\hline Right banks of superior temporal sulcus & 0.030 & n.s. \\
\hline Left supramarginal gyrus & 0.026 & n.s. \\
\hline Right supramarginal gyrus & 0.026 & n.s. \\
\hline Left insula & 0.045 & n.s. \\
\hline Right insula & 0.045 & n.s. \\
\hline Left inferior frontal gyrus (pars opercularis) & 0.026 & n.s. \\
\hline Left inferior frontal gyrus (pars orbitalis) & 0.046 & n.s. \\
\hline Right caudal middle frontal gyrus & 0.026 & n.s. \\
\hline Right superior frontal gyrus & 0.035 & n.s. \\
\hline Right inferior parietal gyrus & 0.031 & n.s. \\
\hline Right superior parietal gyrus & 0.045 & n.s. \\
\hline
\end{tabular}

Abbreviations: $\mathrm{CT}$ = cortical thickness; $\mathrm{GM}=$ gray matter; $\mathrm{HC}=$ healthy control; $\mathrm{n} . \mathrm{s}=$ nonsignificant; RRMS = relapsing-remitting multiple sclerosis. Student $t$ test $p$ values are provided for significant differences. The false discovery rate (FDR) and Holm-Bonferroni corrections for multiple comparisons were applied.

tests by each patient. Thus, patients were classified as cognitively preserved (CP-RRMS; $\mathrm{n}=17$ ) if they had not failed or failed between 0 and 1 tests and as cognitively impaired (CI-RRMS, $\mathrm{n}=$ 18) if they failed 2 or more tests.

Linear regression modeling analyses were performed to identify the contribution of sNfL levels and demographic, clinical, psychiatric, and radiologic variables (including global and regional measures of GM volume and $\mathrm{CT}$ ) as predictors of global neuropsychological status (ZG) scores. Only those demographic and clinical variables that showed a significant correlation $(p<0.05)$ with ZG scores were entered in a stepwise fashion into 2 linear regression models to determine the strongest variable predictors of ZG in our RRMS sample. In addition to those variables that fulfilled this criterion, only MRI variables measures that were statistically different between groups were entered in the regression analysis. Specifically, the first regression model only included global MRI measures, whereas the second model only involved regional MRI measures (volumes and CT). A $p$ value $<0.05$ was considered statistically significant.

\section{Data Availability}

The anonymized raw data analyzed in the present study are available on reasonable request to the corresponding author for purposes of replicating procedures and results.

\section{Results}

\section{Demographic and Clinical Evaluation}

Individuals in both groups were age and sex matched; nevertheless, the HC group had more years of schooling than the RRMS group $(p<0.05)$. Accordingly, all further analyses involving variables of interest were corrected for education level. The RRMS patient sample exhibited low accumulation of disability (EDDS median 1.0) and a few years of disease duration (mean 3.13 years). Table 1 summarizes the main demographic and clinical characteristics of HCs and patients with RRMS.

\section{Neuropsychological Assessment}

Table 2 summarizes neuropsychological results. Notably, there were no significant differences between groups in manipulative IQ. However, patients with RRMS exhibited higher 
Table 5 Correlations Between ZG Scores and Demographic, Clinical, and Radiologic Variables in all Participants and in the RRMS Group

\begin{tabular}{|c|c|c|c|c|}
\hline \multirow[b]{2}{*}{ Variables } & \multicolumn{2}{|c|}{ ZG (all participants) } & \multicolumn{2}{|c|}{ ZG (RRMS group) } \\
\hline & $r, \rho$ & $p$ Values & $r, \rho$ & $p$ Values \\
\hline Age & -0.335 & 0.010 & -0.345 & 0.043 \\
\hline Educational level & 0.419 & 0.001 & 0.372 & 0.028 \\
\hline EDSS & - & - & -0.375 & 0.027 \\
\hline FSS & -0.311 & 0.017 & n.s. & n.s. \\
\hline BDI & -0.383 & 0.003 & -0.365 & 0.031 \\
\hline STAI-S & -0.350 & 0.007 & -0.407 & 0.015 \\
\hline sNfL & -0.346 & 0.008 & -0.336 & 0.047 \\
\hline Left thalamus GM volume & 0.303 & 0.021 & n.s. & n.s. \\
\hline Right thalamus GM volume & 0.280 & 0.034 & n.s. & n.s. \\
\hline Mean CT & 0.455 & 0.000 & 0.337 & 0.048 \\
\hline Right TTG CT & 0.305 & 0.020 & n.s. & n.s. \\
\hline Right STG CT & 0.315 & 0.016 & n.s. & n.s. \\
\hline
\end{tabular}

Abbreviations: BDI = Beck Depression Inventory; CT = cortical thickness = STG = superior temporal gyrus; EDSS = Expanded Disability Status Scale; FSS = Fatigue Severity Scale; GM = gray matter = TTG = transverse temporal gyrus; GM = gray matter; HC = healthy control; $\mathrm{n} . \mathrm{S}$. = nonsignificant; RRMS = relapsingremitting multiple sclerosis; sNfL = serum neurofilament light; STAI-S = State-Trait Anxiety Inventory, State score; ZG = global cognitive Z-score.

Values are expressed as Pearson $(r)$ or Spearman $(\rho)$ coefficients and $p$ values.

scores for fatigue (FSS), depression (BDI), and anxiety (STAI) than HCs. Regarding cognitive measures, patients with RRMS had poorer performance on tests involving both information processing speed/working memory (Paced Auditory Serial Addition Test 3) and verbal episodic memory (including Selective Reminding Test storage, retrieval, and delayed recall scores), corrected for educational level. At the individual level, $25.7 \%$ of patients with RRMS did not fail any BRB-N test, $22.9 \%$ failed 1 test, and $51.4 \%$ failed 2 or more subtests.

\section{Radiologic Results}

Patients with RRMS showed a FLAIR hyperintensity mean lesion volume of $3.77 \pm 5.86 \mathrm{~mL}$. Although no significant differences between groups were observed in global atrophy volume-based measures (BPF and GMF, Table 2), regional analysis of subcortical GM volumes showed a significant reduction in left $(p=0.033)$ and right thalami $(p=0.047)$ in patients with RRMS compared with HCs (Table 3 and Figure 1A). Inverse contrast (patients with RRMS > HCs) did not yield any significant results. Comparing regional cortical volume-based analysis using the Neuromorphometrics atlas between the RRMS and HC groups, there was only 1 significant difference reflected in a reduced GM volume in left inferior frontal orbital frontal gyrus (IFOG) (FDR-adjusted $p=0.031$; Table 4 and Figure 1B).

Regarding surface-based measures, patients with RRMS displayed a significant reduction in global CT compared with HCs
(Table 2). More specifically, regional analysis (following FDR correction) indicated that patients with RRMS exhibited widespread cortical thinning that affected bilateral temporal, parietal, and frontal areas compared with HCs (Table 4 and Figure $1 \mathrm{C}$ for further details). Subsequent conservative analysis (following Holm-Bonferroni correction) retained a significant $\mathrm{CT}$ reduction only in the RRMS group in the right transverse temporal gyrus (TTG, $p=0.037$ ) and right superior temporal gyrus (STG, $p=0.045$ ). Inverse contrast (patients with RRMS $>\mathrm{HCs}$ ) did not yield any significant results.

\section{sNfL Results}

The median (interquartile range) sNfL level was higher in patients with RRMS (38 [31-51]) than in HCs (36 [20-42]). Although patients with RRMS also exhibited increased mean concentrations of sNfL levels, only a statistical trend toward significance was observed $(p=0.087)$ (Table 2).

Regarding the presence or absence of NI, a group effect was observed $\left(\mathrm{F}_{2,55}=3.533 ; p=0.036\right)$. Post hoc tests revealed significantly increased sNfL levels in the CI-RRMS subgroup compared with HCs (Bonferroni-corrected $p=0.036$ ) but not to the CP-RRMS subgroup or between both RRMS subgroups.

\section{sNfL-MRI-Neuropsychological Relationships in Patients With RRMS}

sNfL levels correlated with both the total number of failed tests $(\rho=0.437, p=0.009)$ and with global neuropsychological 
Figure 2 Associations Between Regression Standardized Predicted Values and Global Cognitive Status (ZG Scores) in the RRMS Group

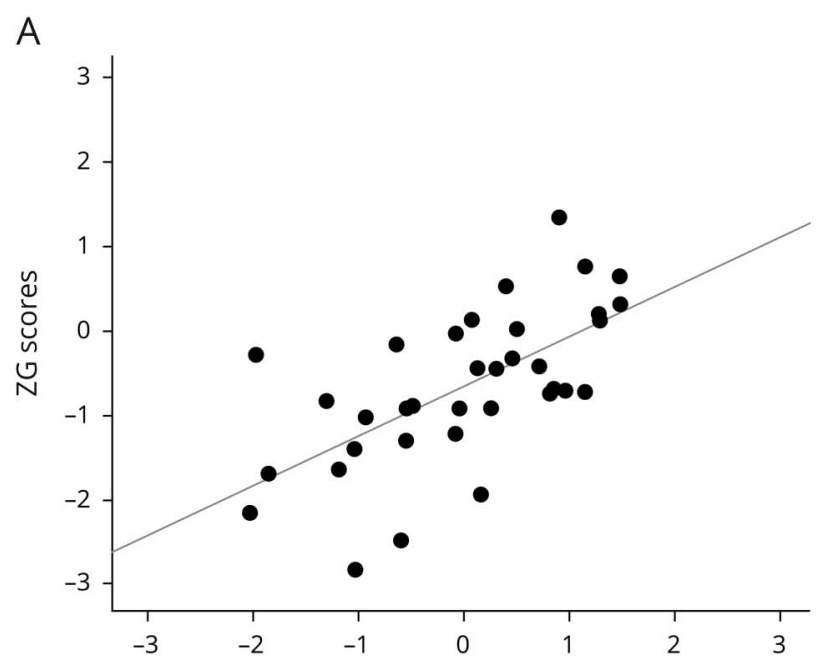

B

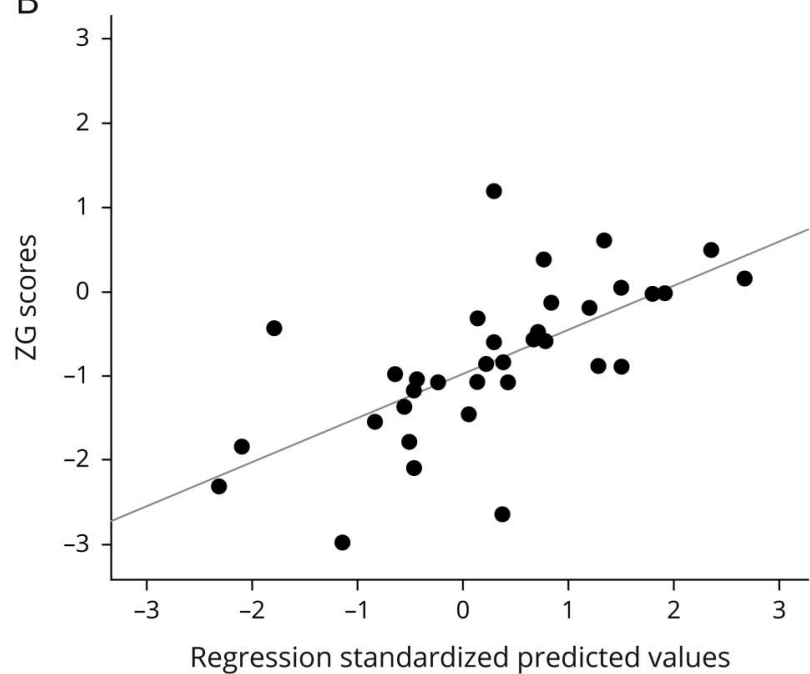

(A) Stepwise regression model retaining STAI state anxiety scores ( $\beta=$ $-0.412, t=-2.969)$, sNfL levels $(\beta=-0.367, t=-2.632)$, and global CT values $\mathrm{CT}(\beta=0.354 ; \mathrm{t}=2.546)$ as significant predictors of global cognitive status (ZG scores) in the RRMS group (adjusted $\mathrm{R}^{2}=0.404 ; p<0.001$ ). (B) Stepwise regression model including regional radiologic variables, retaining STAI state anxiety scores $(\beta=-0.529, \mathrm{t}=-3.751)$, sNfL levels $(\beta=-0.419, \mathrm{t}=$ $-3.018)$ and right TTG thickness $(\beta=0.409 ; t=2.853)$ as significant predictors of global cognitive status (ZG scores) in the RRMS group (adjusted $\mathrm{R}^{2}=0.429$; $p<0.001)$. CT = cortical thickness; RRMS = relapsing-remitting multiple sclerosis; $\mathrm{SNfL}=$ serum neurofilament light chain; TTG = transverse temporal gyrus; ZG = global cognitive Z score.

performance $(r=-0.336, p=0.047)$. Importantly, sNfL levels did not directly correlate with any clinical or radiologic (global/ regional) measure. The results showing significant correlations between ZG scores and other relevant variables are summarized in Table 5.

A first stepwise multiple regression analysis was performed to determine whether candidate variables (age, educational level, EDSS, FSS, BDI, STAI-S, mean CT, and SNfL) predicted ZG scores. This model revealed that STAI-S, sNfL levels, and global $\mathrm{CT}$ were the variables retained in the final model predicting neuropsychological performance $\left(\mathrm{R}^{2}=0.404 ; p<\right.$ 0.001; Figure 2A). To investigate the reliability of regional measures as predictors in cognitive status, a separate stepwise multiple regression analysis was also performed as described above for the same data set of predictive variables and in which the candidate global MRI measures were replaced with regional measures significantly decreased (Holm-Bonferroni corrected) in the RRMS group compared with the HC group (i.e., left/right thalamic GM volumes and CT of the right TTG and STG). This model retained the STAI-S score, sNfL level, and right TTG thickness as variables that best explained neuropsychological performance in patients with RRMS $\left(\mathrm{R}^{2}\right.$ $=0.429 ; p<0.001$; Figure 2B).

\section{Discussion}

In newly diagnosed patients with RRMS, CT and sNfL measurements appear to be promising biomarker combinations for detecting the presence of early cognitive disturbances. In the present study, we demonstrated that the sNfL level alone, as a measure of global neuroaxonal injury, ${ }^{31}$ does not appear to properly differentiate between newly diagnosed patients with RRMS and a healthy population or between patients with RRMS cognitively preserved and displacing signs of NI. However, in combination with global and regional CT measurements, which are considered good direct indices of cortical morphology for detecting neuronal loss or degradation, both biomarkers could reveal 2 parallel pathophysiologic processes underlying the neurodegenerative triggering of MS and predict early cognitive deficits in the most common course of this disease.

A striking result of our study was that sNfL levels, although not observed to significantly differentiate between newly diagnosed patients with RRMS and HCs, were retained in regression models predicting NI in the RRMS group in combination with psychiatric scores and CT measurements. This finding suggests the potential use of sNfL levels as a complementary biomarker of MS cognitive impairment at early stages of the disease and as a relevant measure in clinical settings. Because the neurofilament protein is a major structural component of myelinated axons and is essential to maintain axonal caliber and to facilitate effective nerve conduction, the sNfL level has recently emerged as a useful peripheral blood marker reflecting neuroaxonal damage and in studying neurodegeneration characteristics in MS and other brain-related diseases. Thus, higher plasma and sNfL levels have been associated with faster rates of brain atrophy and cortical neurodegeneration, allowing us to predict disease progression at the early stages of different neurologic conditions. ${ }^{8-11}$

In MS, baseline sNfL concentrations have also been shown to be associated with different measures of structural brain damage, predicting the progression of neurodegeneration and disability in these patients. ${ }^{13,14}$ Importantly, a close association between the temporal dynamics of sNfL levels and brain volume loss over time in normal aging has been recently documented, ${ }^{31}$ suggesting that sNfL levels could further 
reflect posterior-cortical deterioration rather than frontal damage, at least in Parkinson disease. ${ }^{11}$ Likewise, although evidence relating global or regional $\mathrm{CT}$ and $\mathrm{sNfL}$ is indeed scarce, recent studies of patients with presymptomatic Alzheimer disease have demonstrated that sNfL changes are closely associated with both the rate of cortical thinning and cognitive changes, ${ }^{10}$ emphasizing the potential role of the sNfL concentration as a primary marker of neurodegeneration in patients with neurologic diseases and in patients with MS.

Although preliminary evidence for an association between sNfL concentrations and cognitive performance in MS has been recently suggested, this relationship has been exclusively observed in progressive forms of disease thus far. ${ }^{16}$ Therefore, sNfL levels have been suggested to be inconsistently related to neuropsychological performance in MS, raising doubts about their use as a surrogate biomarker of cognitive status in MS. ${ }^{17}$ However, remarkably, our results revealed that reduced sNfL concentrations were associated with better neuropsychological functioning and that their increase was also related to MS patients displaying NI. Furthermore, our data indicate that sNfL levels, in conjunction with CT measurements (rather than volume or atrophy), may contribute to a better explanation of cognitive performance in patients with RRMS. Hence, the combination of multiple neurodegeneration biomarkers, rather than the use of single ones, appears to be a reasonable approach to their improve prognostic value.

Notably, anxiety-state scores, in combination with other biomarkers, also contributed to a prediction of neuropsychological performance in our patient cohort, highlighting the significant effect of anxiety symptoms on cognition in patients with MS. ${ }^{32}$ These findings are not surprising given that high prevalence rates of psychiatric comorbidity ${ }^{33}$ have been well documented in patients with MS and underscore the importance of establishing a psychiatric assessment in patients with MS from initial stages of the disease.

Concerning radiologic measures, we observed no significant differences between patients with RRMS and HCs in global volume-based measures of brain atrophy or GM atrophy (i.e., BPF or GMF, respectively). Importantly, a decreased global CT was found in patients with RRMS compared with HCs, suggesting that at least during the earliest stages of MS, global conventional volume-based global measures may not be sensitive enough to detect subtle structural changes primarily driven by cortical thinning. In this respect, previous studies have documented that FLAIR lesion volumes were associated with global CT reduction in patients with RRMS, indicating that focal inflammatory processes in WM may be related, at least partially, to the progression of cortical atrophy. ${ }^{34,35}$

At the regional level, we demonstrated that GM volume reduction in RRMS was confined to the bilateral thalami. These findings are consistent with previous evidence, which emphasizes thalamic atrophy as an early and sensitive surrogate of neurodegeneration processes in MS. ${ }^{2}$ Although the cause of thalamic atrophy in MS is not fully understood, different studies have demonstrated that thalamic atrophy may be related to a high prevalence of periventricular WM lesions, demyelination or transection of thalamic projection fibers, and subsequent axonal degeneration. ${ }^{36}$ In the RRMS group, the observed decreases in GM volume in the IFOG, a crucial node in the frontoparietal network responsible for cognitive control and emotional processing, were also in line with the findings of previous studies ${ }^{37}$ and has been linked to the presence of mood disturbances in patients with MS. ${ }^{38}$ Furthermore, although this volume-based approach did not reveal any other significant cortical GM volume reduction in our MS group, regional analysis of CT revealed a significant thinning of right temporal areas, specifically affecting the TTG and STG in the right hemisphere. These findings are also in agreement with those of a previous report on a similar cohort of recently diagnosed and mildly disabled patients with RRMS. ${ }^{4}$ Notably, these differences were overlooked using traditional volume-based methodology, suggesting that this approach may not be sensitive enough to detect regional cortical changes occurring during the first stages of MS. Of note, 2 different atlases were used to obtain regional cortical GM volumes and CT values. Using a single atlas to quantify these 2 MRI measures is not straightforward because there are substantial technical impediments to combine vertices/voxels-based atlases. Hence, we cannot completely confirm that the discrepancies found between regional GM volume and $\mathrm{CT}$ were due to the different cortical parcellation methods implemented.

Although the cortical thinning in the temporal lobe was more significant over the right hemisphere in our RRMS patient sample, thinning of the left temporal cortex, as well as CT reductions of parietal and frontal regions (Figure 1C), was also evident after a less conservative multiple comparisons correction, demonstrating the relevance of this common finding. Of interest, an association between temporal lobe thinning and lower cognitive performance has been previously demonstrated in patients with RRMS. ${ }^{5,39,40}$ In this association, STG thinning has been directly linked to poorer performance in tasks assessing visuospatial memory, attention, and information processing speed. ${ }^{5,40}$ Remarkably, in our present study, although global CT was also a significant predictor of cognitive status in the RRMS group, replacing this measure with regional right TTG thinning slightly increased the predictive value of the model and therefore accounted for greater variance in explaining cognitive deficits than global CT.

Accumulating evidence suggests that temporal lobe structural changes represent an important feature over the entire disease course of MS. In this regard, the temporal GM volume loss rate has been suggested to be a surrogate marker for the transition from the relapsing-remitting to the secondary progressive (SP) phase of the disease. ${ }^{3,35}$ Likewise, cortical thinning in temporal regions has been previously described in newly diagnosed and mildly disabled patients with RRMS, 
affecting the left hemisphere more severely. ${ }^{4,6}$ Furthermore, the TTG, as a primary area on the superior surface of the temporal lobe, appears to play a role in processing information related to auditory and language input and speech understanding. However, the TTG has also been implicated in higher-order cognitive functions and in receiving top-down influences of attention, such as in the processing of speechrelated cues that facilitate learning and perceiving new speech sounds ${ }^{41}$ and in memory mechanisms underlying stimulus comparisons. ${ }^{42}$ Hence, our results underscore that specific key brain regions involved in a wide variety of distributed cognitive processes appear to display a well-defined cortical thinning pattern. In this pattern, temporal lobe thinning, probably due to being adjacent to neocortical association areas, could be a potential fingerprint of underlying MS pathology from early stages of the disease. Both the distribution pattern and the predominant site of occurrence of the damaging WM lesions observed in MS, which evolves around the horns of the ventricles within fiber connections and are particularly noted in the frontal and temporal cortical areas, ${ }^{43}$ may lead to remote retrograde changes that would result in the subsequent diminished thickness of the TTG and other temporal brain areas. Consequently, this damage may contribute to impairments of phonologic encoding, working memory, and verbal processing that are often prominent in these patients.

The present study had some limitations. First, we recruited a clinical sample composed exclusively of recently diagnosed and mildly disabled patients with RRMS. A homogenous sample is often desirable in several studies; however, our conclusions are not applicable in progressive subtypes of MS, such as those with SP or the primary progressive subtype. Second, it can be considered another limitation that the overall age range of our MS cohort was large, which was due to the late onset of some patients (11\%) diagnosed after age 50 years. Accumulating evidence indicates that late-onset MS prevalence/diagnoses have increased in recent decades. However, although our patients with MS were similar in many characteristics (e.g., low EDSS scores), a more homogenous age range would have been desirable to minimize the possible impact of age. Third, a full characterization of cortical GM damage in MS should include cortical lesions, which would have improved the potential scope of our MRI assessment and conclusions. Finally, the potential value of the sNfL concentration as a marker of neuroaxonal injury in MS should consider the possible treatment effect ${ }^{13}$ because its reduction over time or between patients and HCs could represent regression to the mean as a consequence of disease-modifying treatments at the time of the assessment. In this respect, although all measures were collected in a reasonable short time period, a less variable intermeasurement interval would have also been preferable to limit the impact that this could have in early MS. However, none of the patients with RRMS included in our study had a relapse or other significant clinical event between neuropsychological/blood sample testing and MRI examination.
In summary, the results of the present study indicated that sNfL levels reflect cortical neurodegeneration in recently diagnosed in patients with RRMS. In particular, the association of sNfL levels with cognitively impaired patients with MS suggests the mirroring of pathologic mechanisms likely involved in the development of MS progression that, in addition to the thinning of key cortical regions (rather that to regional brain volume measures), would be associated with the presence cognitive dysfunction in early stages of the disease, even in patients with minimal physical disability. Based on these findings, and in accordance with recent new evidence, we postulate that the combination of biomarkers of both cortical macro- (cortical thinning of temporal regions) and microstructural (increased sNfL level) brain damage may most closely reflect subtle neurodegeneration changes and a strong association with the presence of first cognitive deficits in newly diagnosed patients with RRMS.

\section{Acknowledgment}

The authors thank the MS unit research staff and the Neuroradiology Department from the Puerta del Mar University Hospital for their assistance with recruitment and data collection. The authors especially want to thank patients, their families, and healthy participants for their collaboration.

\section{Study Funding}

This work was supported by the European Regional Development Fund and the Spanish Ministry of Science, Innovation and Universities (grant: RTI2018-096951-A-I00) and the Ministry of Economy and Competitiveness (grant: RYC-2015-18467).

\section{Disclosure}

A.J. Cruz-Gomez and J.D. Gomez-Ramirez were supported by 2 postdoctoral fellowships from the Department of Health of the Andalusia Health Service (grants: PI-00252017 and PI-0034-2019). L Forero received speaker fees and travel support from Biogen, Merck Serono, Novartis, Sanofi, Teva, and Roche. E Lozano-Soto and F Sanmartino were supported by the University of Cadiz through 2 predoctoral fellowships (UCA-PIF program). F Cano-Cano was supported by the INiBICA through a predoctoral fellowship. R. Rashid-Lopez was supported by a clinical researcher fellowship (grant: PI-0025-2017) and received speaker fees and travel support from Teva, AbbVie, Zambon, BIAL, and Italfarmaco. J. Paz-Esposito had nothing to disclose. R. Espinosa-Rosso received speaker fees and travel support from Teva, AbbVie, Zambon, BIAL, Italfarmaco, Biogen, Merck Serono, Novartis, Sanofi, and Roche. J.J. GonzalezRosa was supported by a tenure-track "Ramon y Cajal" research fellowship/grant (RYC-2015-18467). Go to Neurology.org/ $\mathrm{NN}$ for full disclosures

\section{Publication History}

Received by Neurology: Neuroimmunology \& Neuroinflammation February 18, 2021. Accepted in final form July 13, 2021. 
Appendix Authors

\begin{tabular}{|c|c|c|}
\hline Name & Location & Contribution \\
\hline $\begin{array}{l}\text { Álvaro Javier } \\
\text { Cruz-Gómez }\end{array}$ & $\begin{array}{l}\text { Institute of Biomedical } \\
\text { Research and Innovation of } \\
\text { Cadiz (INiBICA), Cadiz, Spain. } \\
\text { Psychology Department, } \\
\text { University of Cadiz, Spain. }\end{array}$ & $\begin{array}{l}\text { Design and } \\
\text { conceptualization of the } \\
\text { study; neuropsychological } \\
\text { assessment; MRI } \\
\text { preprocessing and análysis; } \\
\text { statistical analysis of } \\
\text { radiologic, } \\
\text { neuropsychological, clinical, } \\
\text { and sNfL data; and drafting } \\
\text { the manuscript for } \\
\text { intellectual content. }\end{array}$ \\
\hline
\end{tabular}

\begin{tabular}{lll}
\hline $\begin{array}{l}\text { Lucía Forero, } \\
\text { MD }\end{array}$ & $\begin{array}{l}\text { Institute of Biomedical } \\
\text { Research and Innovation of } \\
\text { Cadiz (INiBICA), Cadiz, Spain. } \\
\text { Neurology Department, } \\
\text { Puerta del Mar University } \\
\text { Hospital, Cadiz, Spain. }\end{array}$ & $\begin{array}{l}\text { Patient recruitment; clinical } \\
\text { assessment; and design and } \\
\text { conceptualization of the } \\
\text { study. }\end{array}$ \\
\hline $\begin{array}{lll}\text { Elena } \\
\text { Lozano-Soto, } \\
\text { BSc }\end{array}$ & $\begin{array}{l}\text { Institute of Biomedical } \\
\text { research and Innovation of } \\
\text { Cadiz (INiBICA), Cadiz, Spain. } \\
\text { Psychology Department, }\end{array}$ & $\begin{array}{l}\text { Neuropsychological } \\
\text { assessment and analysis of } \\
\text { neuropsychological and } \\
\text { clinical data. }\end{array}$ \\
\hline $\begin{array}{l}\text { University of Cadiz, Spain. } \\
\text { Cano-Cano, }\end{array}$ & $\begin{array}{l}\text { Institute of Biomedical } \\
\text { research and Innovation of } \\
\text { BSc }\end{array}$ & $\begin{array}{l}\text { Acquisition, storage, and } \\
\text { analysis of blood samples to } \\
\text { obtain serum neurofilament }\end{array}$ \\
& & $\begin{array}{l}\text { light chain (sNf) } \\
\text { concentrations. }\end{array}$
\end{tabular}

\begin{tabular}{lll}
\hline Florencia & Institute of Biomedical & Analysis of radiologic, \\
Sanmartino, & Research and Innovation of & neuropsychological, clinical, \\
BSc & Cadiz (INiBICA), Cadiz, Spain. & and sNfL data. \\
& Psychology Department, & \\
& University of Cadiz, Spain.
\end{tabular}

Raúl Rashid- Institute of Biomedical Patient recruitment and

López, MD Research and Innovation of clinical assessment. Cadiz (INiBICA), Cadiz, Spain.

Neurology Department, Puerta del Mar University Hospital, Cadiz, Spain.

\begin{tabular}{|c|c|c|}
\hline $\begin{array}{l}\text { Jaime D. } \\
\text { Gómez- } \\
\text { Ramirez, } \\
\text { PhD }\end{array}$ & $\begin{array}{l}\text { Institute of Biomedical } \\
\text { research and Innovation of } \\
\text { Cadiz (INiBICA), Cadiz, Spain. } \\
\text { Psychology Department, } \\
\text { University of Cadiz, Spain. }\end{array}$ & $\begin{array}{l}\text { MRI preprocessing and MRI } \\
\text { analysis. }\end{array}$ \\
\hline
\end{tabular}

\begin{tabular}{lll}
\hline José Paz & Radiodiagnostic & MRI protocol planning; \\
Expósito, & Department, Puerta del Mar & acquisition of MRI sequences \\
MD, PhD & Hospital, Cadiz. Spain. & $\begin{array}{l}\text { of participants; and drafting } \\
\text { the manuscript for } \\
\text { intellectual content. }\end{array}$ \\
& &
\end{tabular}

Raúl Institute of Biomedical Patient recruitment; clinical

Espinosa- Research and Innovation of assessment; and drafting the

Rosso, MD Cadiz (INiBICA), Cadiz, Spain. manuscript for intellectual Neurology Department, content.

Puerta del Mar University Hospital, Cadiz, Spain.

\begin{tabular}{lll}
\hline Javier J. & Institute of Biomedical & Design and \\
González- & Research and Innovation of & conceptualization of the \\
Rosa, PhD & Cadiz (INiBICA), Cadiz, Spain. & study; analysis of radiologic, \\
& Psychology Department, & neuropsychological, clinical, \\
University of Cadiz, Spain. & $\begin{array}{l}\text { and sNfL data; interpretation } \\
\text { of the data; and drafting and } \\
\text { revision of the manuscript } \\
\text { for intellectual content. }\end{array}$ \\
& &
\end{tabular}

\section{References}

1. Filippi M, Rocca MA. MR imaging of gray matter involvement in multiple sclerosis: implications for understanding disease pathophysiology and monitoring treatment efficacy. AJNR Am J Neuroradiol. 2010;31(7):1171-1177.
2. Azevedo CJ, Cen SY, Khadka S, et al. Thalamic atrophy in multiple sclerosis: a magnetic resonance imaging marker of neurodegeneration throughout disease. Ann Neurol. 2018;83(2):223-234.

3. Eshaghi A, Prados F, Brownlee WJ, et al. Deep gray matter volume loss drives disability worsening in multiple sclerosis. Ann Neurol. 2018;83(2):210-222.

4. Narayana PA, Govindarajan KA, Goel P, et al. Regional cortical thickness in relapsing remitting multiple sclerosis: a multi-center study. Neuroimage Clin. 2013;2: 120-131.

5. Nygaard GO, Walhovd KB, Sowa $\mathrm{P}$, et al. Cortical thickness and surface area relate to specific symptoms in early relapsing-remitting multiple sclerosis. Mult Scler J. 2015; 21(4):402-414.

6. Calabrese M, Rinaldi F, Mattisi I, et al. Widespread cortical thinning characterizes patients with MS with mild cognitive impairment. Neurology. 2010;74(4): 321-328.

7. Lemaitre H, Goldman AL, Sambataro F, et al. Normal age-related brain morphometric changes: nonuniformity across cortical thickness, surface area and gray matter volume? Neurobiol Aging. 2012;33(3):617.e1-617.e9.

8. Matías-Guiu JA, Gómez-Pinedo U, Forero L, et al. Plasma neurofilament light chain in primary progressive aphasia and related disorders: clinical significance and metabolic correlates. J Alzheimers Dis. 2019;72(3):773-782.

9. Rohrer JD, Woollacott IO, Dick KM, et al. Serum neurofilament light chain protein is a measure of disease intensity in frontotemporal dementia. Neurology. 2016;87(13): 1329-1336.

10. Preische O, Schultz SA, Apel A, et al. Serum neurofilament dynamics predicts neurodegeneration and clinical progression in presymptomatic Alzheimer's disease. Nat Med. 2019;25(2):277-283.

11. Sampedro F, Pérez-González R, Martínez-Horta S, Marín-Lahoz J, Pagonabarraga J, Kulisevsky J. Serum neurofilament light chain levels reflect cortical neurodegeneration in de novo Parkinson's disease. Parkinsonism Relat Disord. 2020;74:43-49.

12. Disanto G, Barro C, Benkert P, et al. Serum Neurofilament light: a biomarker of neuronal damage in multiple sclerosis. Ann Neurol. 2017;81(6):857-870.

13. Kuhle J, Kropshofer H, Haering DA, et al. Blood neurofilament light chain as a biomarker of MS disease activity and treatment response. Neurology. 2019;92(10) e1007-e1015.

14. Chitnis T, Gonzalez C, Healy BC, et al. Neurofilament light chain serum levels correlate with 10-year MRI outcomes in multiple sclerosis. Ann Clin Transl Neurol. 2018;5(12):1478-1491.

15. Uher T, Schaedelin S, Srpova B, et al. Monitoring of radiologic disease activity by serum neurofilaments in MS. Neurol Neuroimmunol Neuroinflamm. 2020;7(4):e714.

16. Kalatha T, Arnaoutoglou M, Koukoulidis $\mathrm{T}$, et al. Does cognitive dysfunction correlate with neurofilament light polypeptide levels in the CSF of patients with multiple sclerosis? J Int Med Res. 2019;47(5):2187-2198.

17. Aktas O, Renner A, Huss A, et al. Serum neurofilament light chain: no clear relation to cognition and neuropsychiatric symptoms in stable MS. Neurol Neuroimmunol Neuroinflammation. 2020;7(6):e885.

18. Barro C, Benkert P, Disanto G, et al. Serum neurofilament as a predictor of disease worsening and brain and spinal cord atrophy in multiple sclerosis. Brain. 2018;141(8): 2382-2391.

19. Polman CH, Reingold SC, Banwell B, et al. Diagnostic criteria for multiple sclerosis: 2010 revisions to the McDonald criteria. Ann Neurol. 2011;69(2):292-302.

20. Oldfield RC. The assessment and analysis of handedness: the Edinburgh inventory. Neuropsychologia. 1971;9(1):97-113.

21. Kurtzke JF. Rating neurologic impairment in multiple sclerosis: an expanded disability status scale (EDSS). Neurology. 1983;33(11):1444-1452.

22. Sepulcre J, Vanotti $S$, Hernández R, et al. Cognitive impairment in patients with multiple sclerosis using the Brief Repeatable Battery-Neuropsychology test. Mult Scler. 2006;12(2):187-195.

23. Wechsler D. Wechsler Adult Intelligence Scale III. TEA; 2001.

24. Krupp LB, LaRocca NG, Muir-Nash J, Steinberg AD. The fatigue severity scale. Application to patients with multiple sclerosis and systemic lupus erythematosus. Arch Neurol. 1989;46(10):1121-1123.

25. Wideman TH, Sullivan MJL, Inada S, et al. Beck depression inventory (BDI). In: Encyclopedia of Behavioral Medicine. Springer New York; 2013:178-179.

26. Spielberger CD, Gorsuch RL, Lushene R, Vagg PR, Jacobs GA. Manual for the StateTrait Anxiety Inventory. Consulting Psychologists Press; 1983.

27. Gelineau-Morel R, Tomassini V, Jenkinson M, Johansen-Berg H, Matthews PM, Palace J. The effect of hypointense white matter lesions on automated gray matter segmentation in multiple sclerosis. Hum Brain Mapp. 2012;33(12):2802-2814.

28. Patenaude B, Smith SM, Kennedy DN, Jenkinson M. A Bayesian model of shape and appearance for subcortical brain segmentation. Neuroimage. 2011;56(3):907-922.

29. Dahnke R, Yotter RA, Gaser C. Cortical thickness and central surface estimation. Neuroimage. 2013;65:336-348.

30. Desikan RS, Ségonne F, Fischl B, et al. An automated labeling system for subdividing the human cerebral cortex on MRI scans into gyral based regions of interest. Neuroimage. 2006;31(3):968-980.

31. Khalil M, Pirpamer L, Hofer E, et al. Serum neurofilament light levels in normal aging and their association with morphologic brain changes. Nat Commun. 2020; 11(1):812

32. Vissicchio NA, Altaras C, Parker A, et al. Relationship between anxiety and cognition in multiple sclerosis. Int J MS Care. 2019;21(4):151-156.

33. Boeschoten RE, Braamse AMJ, Beekman ATF, et al. Prevalence of depression and anxiety in Multiple Sclerosis: a systematic review and meta-analysis. J Neurol Sci. 2017, $37: 331-341$ 
34. Bergsland $\mathrm{N}$, Laganà $\mathrm{MM}$, Tavazzi $\mathrm{E}$, et al. Corticospinal tract integrity is related to primary motor cortex thinning in relapsing-remitting multiple sclerosis. Mult Scler J. 2015;21(14):1771-1780.

35. Tsagkas C, Chakravarty MM, Gaetano L, et al. Longitudinal patterns of cortical thinning in multiple sclerosis. Hum Brain Mapp. 2020;41(8):2198-2215.

36. Mahajan KR, Nakamura K, Cohen JA, Trapp BD, Ontaneda D. Intrinsic and extrinsic mechanisms of thalamic pathology in multiple sclerosis. Ann Neurol. 2020;88(1):81-92.

37. Audoin B, Zaaraoui W, Reuter F, et al. Atrophy mainly affects the limbic system and the deep grey matter at the first stage of multiple sclerosis. J Neurol Neurosurg Psychiatry. 2010;81(6):690-695.

38. Pfaff L, Lamy J, Noblet V, et al. Emotional disturbances in multiple sclerosis: a neuropsychological and fMRI study. Cortex. 2019;117:205-216.
39. Tillema JM, Hulst HE, Rocca MA, et al. Regional cortical thinning in multiple sclerosis and its relation with cognitive impairment: a multicenter study. Mult Scler. 2016; 22(7):901-909.

40. Achiron A, Chapman J, Tal S, Bercovich E, Gil H, Achiron A. Superior temporal gyrus thickness correlates with cognitive performance in multiple sclerosis. Brain Struct Funct. 2013;218(4):943-950.

41. Warrier C, Wong P, Penhune V, et al. Relating structure to function: Heschl's gyrus and acoustic processing. J Neurosci. 2009;29(1):61-69.

42. Opitz B, Schröger E, von Cramon DY. Sensory and cognitive mechanisms for pre attentive change detection in auditory cortex. Eur J Neurosci. 2005;21(2):531-535.

43. Sailer M, Fischl B, Salat D, et al. Focal thinning of the cerebral cortex in multiple sclerosis. Brain. 2003;126(pt 8):1734-1744. 


\title{
Neurology \\ Neuroimmunology \& Neuroinflammation
}

\author{
Cortical Thickness and Serum NfL Explain Cognitive Dysfunction in Newly Diagnosed \\ Patients With Multiple Sclerosis \\ Álvaro J. Cruz-Gomez, Lucía Forero, Elena Lozano-Soto, et al. \\ Neurol Neuroimmunol Neuroinflamm 2021;8; \\ DOI 10.1212/NXI.0000000000001074
}

This information is current as of August 31, 2021

\section{Updated Information \& \\ Services}

References

Subspecialty Collections

Permissions \& Licensing

Reprints including high resolution figures, can be found at:

http://nn.neurology.org/content/8/6/e1074.full.html

This article cites 40 articles, 4 of which you can access for free at: http://nn.neurology.org/content/8/6/e1074.full.html\#\#ref-list-1

This article, along with others on similar topics, appears in the following collection(s):

MRI

http://nn.neurology.org//cgi/collection/mri

Multiple sclerosis

http://nn.neurology.org//cgi/collection/multiple_sclerosis

Neuropsychological assessment

http://nn.neurology.org//cgi/collection/neuropsychological_assessment Volumetric MRI

http://nn.neurology.org//cgi/collection/volumetric_mri

Information about reproducing this article in parts (figures,tables) or in its entirety can be found online at:

http://nn.neurology.org/misc/about.xhtml\#permissions

Information about ordering reprints can be found online:

http://nn.neurology.org/misc/addir.xhtml\#reprintsus

Neurol Neuroimmunol Neuroinflamm is an official journal of the American Academy of Neurology.

Published since April 2014, it is an open-access, online-only, continuous publication journal. Copyright

Copyright $\odot 2021$ The Author(s). Published by Wolters Kluwer Health, Inc. on behalf of the American

Academy of Neurology.. All rights reserved. Online ISSN: 2332-7812.

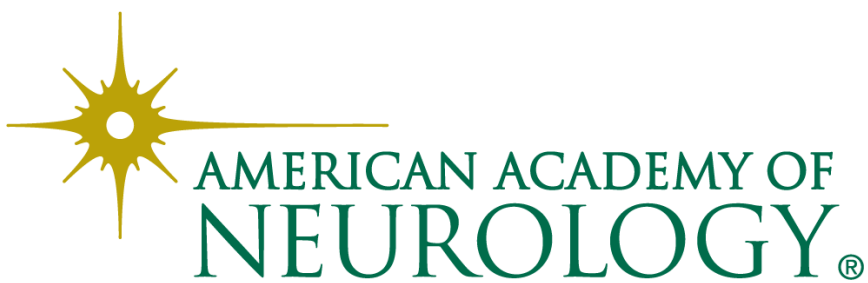

